\title{
Congruent Numbers and The Rank of Elliptic Curves
}

\author{
Yuanbo Liu ${ }^{1, \text { a }}$ \\ ${ }^{1}$ Qingdao University, Shandong Province ,China \\ liuyuanbo15@126.com
}

Keywords: Non-congruent number; Congruent number; Elliptic Curves

Abstract. Let $p$ and $q$ be prime with $p \equiv 3(\bmod 8)$ and $q \equiv 5(\bmod 8)$, and let $\left(\frac{p}{q}\right)=-1$. If $n=p q$, then $n$ is a congruent if and only if the equation $2 q w^{2}=1+p^{2} z^{4}$ has rational solutions. And The Birch Swinnerton-Dyer conjecture predicts that the rank of $E_{n}(Q)$ is one.

\section{Introduction}

If $n=\frac{1}{2} a b$ with rational numbers $a, b$ being two right sides of a rational right triangle, the $n$ is a congruent number. Otherwise $n$ is a non-congruent number. The elliptic curve has an equation $E_{n}: y^{2}=x^{3}-n^{2} x$, then $n$ is a congruent if and only if $E_{n}(Q)$ has a non-zero rank. The problem of congruent number is very old and Arab scholars discussed it in the tenth century ${ }^{[1]}$.

The Birch Swinnerton-Dyer conjecture predicts that if $n \equiv 5,6 \operatorname{or} 7(\bmod 8)$ it is a congruent ${ }^{[2]}$. The conjecture also made by Alter, Curtz and Kubota ${ }^{[3]}$.

In this paper we discuss $n=p q$ with $p \equiv 3(\bmod 8)$ and $q \equiv 5(\bmod 8)$.

\section{2-isogeny}

Let $E / Q$ and $E^{\prime} / Q$ be Elliptic curves given respectively by the equations

$$
E_{n}: y^{2}=x^{3}-n^{2} x \text { and } E_{n}^{\prime}: y^{2}=x^{3}+4 n^{2} x
$$

and let

$$
\phi: E \rightarrow E^{\prime}, \phi(x, y)=\left(y^{2} / x^{2}, y\left(x^{2}+n^{2}\right) / x^{2}\right)
$$

be the isogeny of degree 2 with kernel $E[\phi]=\{o,(0,0)\}$.

Then there is an exact sequence

$$
\begin{aligned}
0 \rightarrow E^{\prime}(Q) / \phi(E(Q)) & \rightarrow Q(S, 2) \rightarrow W C(E / Q) \\
(X, Y) & \mapsto X \quad, d \mapsto\left\{C_{d} / Q\right\} \\
o & \mapsto 1 \\
(0,0) & \mapsto-1
\end{aligned}
$$

where $S=\{\infty\} \cup\{p \mid 2 n\}$ and $Q(S, 2)=\left\{b \in Q^{* 2} / Q^{*}: \operatorname{ord}_{p}(b) \equiv 0(\bmod 2), \forall p \notin S\right\}$.

Furthermore, for each $d \in Q(S, 2)$, let $C_{d} / Q$ be the homogeneous space for $E / Q$ by the equation $C_{d}: d w^{2}=d^{2}+4 n^{2} z^{4}$. Then the $\phi$-Selmer group is

$$
S^{(\phi)}(E / Q) \cong\left\{d \in Q(S, 2): C_{d}\left(Q_{p}\right) \neq \varnothing, \forall p \in S\right\} .
$$

Finally, we have the map 


$$
\varphi: C_{d} \rightarrow E^{\prime}, \varphi(z, w)=\left(\frac{d}{z^{2}},-\frac{d w}{z^{3}}\right) \cdot(* * *)
$$

Let $\hat{\phi}: E^{\prime} \rightarrow E$ be the dual of $\phi$ so that $\phi \hat{\phi}=[2]$ and $\hat{\phi} \phi=[2]$. Let $C_{d}^{\prime} / Q$ be the homogeneous space for $E^{\prime} / Q$ by the equation $C_{d}^{\prime}: d w^{2}=d^{2}-n^{2} z^{4}$.Then the $\hat{\phi}$-Selmer group is

$$
S^{(\hat{\phi})}\left(E^{\prime} / Q\right) \cong\left\{d \in Q(S, 2): C_{d}^{\prime}\left(Q_{p}\right) \neq \varnothing, \forall p \in S\right\} .
$$

Moreover, we have the following exact sequences:

$$
0 \rightarrow \frac{E^{\prime}(Q)[\hat{\phi}]}{\phi(E(Q)[2])} \rightarrow \frac{E^{\prime}(Q)}{\phi(E(Q))} \rightarrow \frac{E(Q)}{2 E(Q)} \rightarrow \frac{E(Q)}{\hat{\phi}\left(E^{\prime}(Q)\right)} \rightarrow 0
$$

\section{2. $n=p q$}

Lemma 1. Let $n=p q$ with $p \equiv 3(\bmod 8)$ and $q \equiv 5(\bmod 8)$, and let $\left(\frac{p}{q}\right)=-1$. Then the $\hat{\phi}$-Selmer group $S^{(\hat{\phi})}\left(E^{\prime} / Q^{\prime}\right)=\{ \pm 1, \pm n\}$, and $\frac{E(Q)}{\hat{\phi}\left(E^{\prime}(Q)\right)}=\{o,(0,0),( \pm n, 0)\}$.

Proof. We will use the exact sequence $\left(^{*}\right)$ to compute $\frac{E(Q)}{\hat{\phi}\left(E^{\prime}(Q)\right)}$. Now $S=\{2, p, q, \infty\}$ and

$$
Q(S, 2)=\{ \pm 1, \pm 2, \pm p, \pm q, \pm 2 p, \pm 2 q, \pm p q, \pm 2 p q\} .
$$

For $d \in Q(S, 2), C_{d}^{\prime}$ has the equation

$$
C_{d}^{\prime}: d w^{2}=d^{2}-p^{2} q^{2} z^{4} .
$$

Claim: For each $d \in Q(S, 2), d \neq \pm 1, \pm n$, there exist $p \in S$ such that $C_{d}^{\prime}\left(Q_{p}\right)=\varnothing$.

1) $d= \pm p, \quad C_{ \pm p}^{\prime}: \pm p w^{2}=p^{2}-p^{2} q^{2} z^{4}$. Let $\left(\frac{z^{\prime}}{t}, \frac{w^{\prime}}{t^{2}}\right)=(p z, p w)$ with $\left(z^{\prime}, w^{\prime}, t\right) \in Z$, then $C_{d}^{\prime}: p w^{\prime 2}=t^{4}-q^{2} z^{\prime 4}$. Since $q \mid t$ if and only if $q \mid w^{\prime}$, Then suppose $\left(z^{\prime}, w^{\prime}, t\right) \in C_{ \pm p}^{\prime}\left(Q_{q}\right)$ with $0 \neq\left(z^{\prime}, w^{\prime}, t\right) \in Z_{p}$ and $\operatorname{ord}_{q} w^{\prime}=0$. We have $\left(\frac{ \pm p}{q}\right)=1$, then $\left(\frac{p}{q}\right)=1$ which contradicts $\left(\frac{p}{q}\right)=-1$. Therefore $C_{ \pm p}^{\prime}\left(Q_{q}\right)=\varnothing$.

2) $d=2 d^{\prime}, d^{\prime} \in Q(S, 2)$ and $\operatorname{ord}_{2} d=0, C_{d}^{\prime}: 2 d^{\prime} w^{\prime 2}=4 d^{\prime 2} t^{4}-p^{2} q^{2} z^{\prime 4}$. Then $\operatorname{ord}_{2} 2 p w^{\prime 2}=1+2 k_{1}$, $\operatorname{ord}_{2} 4 d^{\prime} t^{4}=2+4 k_{2}$ and $\operatorname{ord}_{2}\left(p^{2} q^{2} z^{\prime 4}\right)=4 k_{3}$. Therefore $C_{2 d}^{\prime}\left(Q_{2}\right)=\varnothing$.

3) $d= \pm q, \quad C_{ \pm q}^{\prime}: \pm q w^{2}=q^{2}-p^{2} q^{2} z^{4}$. Let $\left(\frac{z^{\prime}}{t}, \frac{w^{\prime}}{t^{2}}\right)=(q z, q w) \quad$ with $\quad\left(z^{\prime}, w^{\prime}, t\right) \in Z$, then $C_{d}^{\prime}: q w^{\prime 2}=t^{4}-p^{2} z^{\prime 4}$. Since $q \mid t$ if and only if $q \mid z^{\prime}$. Then suppose $\left(z^{\prime}, w^{\prime}, t\right) \in C_{ \pm p}^{\prime}\left(Q_{q}\right)$ with $0 \neq\left(z^{\prime}, w^{\prime}, t\right) \in Z_{p}$ and $\operatorname{ord}_{q} z^{\prime} t=0$. We have $\left(\frac{p^{2}}{q}\right)_{4}=1$, then $\left(\frac{ \pm p}{q}\right)=1$ which contradicts $\left(\frac{p}{q}\right)=-1$. Therefore $C_{ \pm q}^{\prime}\left(Q_{q}\right)=\varnothing$.

This completes the proof of claim so we have $S^{(\hat{\phi})}\left(E^{\prime} / Q^{\prime}\right)=\{ \pm 1, \pm n\}$. Then $\frac{E(Q)}{\hat{\phi}\left(E^{\prime}(Q)\right)}=\{o,(0,0),( \pm n, 0)\}$, since $\{o,(0,0),( \pm n, 0)\} \in \frac{E(Q)}{\hat{\phi}\left(E^{\prime}(Q)\right)}$. 
Lemma 2. Let $n=p q$ with $p \equiv 3(\bmod 8)$ and $q \equiv 5(\bmod 8)$, and let $\left(\frac{p}{q}\right)=-1$. Then the $\phi$-Selmer group $S^{(\phi)}(E / Q) \in\{1,2 q\}$, and $\left|\frac{E^{\prime}(Q)}{\phi(E(Q))}\right| \leq 2$.

Proof . We also use the exact sequence $\left(^{*}\right)$ to compute $\frac{E^{\prime}(Q)}{\phi(E(Q))}$. Now $S=\{2, p, q, \infty\}$ and

$$
Q(S, 2)=\{ \pm 1, \pm 2, \pm p, \pm q, \pm 2 p, \pm 2 q, \pm p q, \pm 2 p q\} .
$$

For $d \in Q(S, 2), C_{d}$ has the equation

$$
C_{d}: d w^{2}=d^{2}+4 p^{2} q^{2} z^{4} .
$$

Claim: For each $d \in Q(S, 2), d \neq 1,2 p$, there exist $p \in S$ such that $C_{d}\left(Q_{p}\right)=\varnothing$.

1) $C_{d}\left(Q_{\infty}\right)=\varnothing \Leftrightarrow d<0$. Then $d>0$.

2) $d=q, p \quad, \quad C_{d}: d w^{2}=d^{2}+4 p^{2} q^{2} z^{4}$. Let $\left(\frac{z^{\prime}}{t}, \frac{w^{\prime}}{t^{2}}\right)=(d z, d w) \quad$ with $\quad\left(z^{\prime}, w^{\prime}, t\right) \in Z$, then $C_{d}: d w^{\prime 2}=t^{4}+4 \frac{n^{2}}{d^{2}} z^{\prime 4}$. Since $\frac{n}{d} \mid t$ if and only if $\frac{n}{d} \mid w^{\prime}$, then suppose $\left(z^{\prime}, w^{\prime}, t\right) \in C_{d}\left(Q_{\frac{n}{d}}\right)$ with $0 \neq\left(z^{\prime}, w^{\prime}, t\right) \in Z_{\frac{n}{d}}$ and $\operatorname{ord}_{q} w^{\prime}=0$. We have $\left(\frac{d}{n / d}\right)=1$, which contradicts $\left(\frac{p}{q}\right)=-1$. Therefore $C_{d}\left(Q_{\frac{n}{d}}\right)=\varnothing$

3) $p \mid d, C_{d}: d w^{\prime 2}=t^{4}+4 \frac{n^{2}}{d^{2}} z^{\prime 4}$ with $\left(z^{\prime}, w^{\prime}, t\right) \in Z$. Since $p \mid t$ if and only if $p \mid z^{\prime}$, then suppose $\left(z^{\prime}, w^{\prime}, t\right) \in C_{d}\left(Q_{p}\right)$ with $0 \neq\left(z^{\prime}, w^{\prime}, t\right) \in Z_{p}$ and $\operatorname{ord}_{q} t z^{\prime}=0$. Then $\left(\frac{-1}{p}\right)=1$, which contradicts $p \equiv 3(\bmod 8)$.

This completes the proof of claim so we have $\phi$-Selmer group $S^{(\phi)}(E / Q) \in\{1,2 q\}$. Then $\left|\frac{E^{\prime}(Q)}{\phi(E(Q))}\right| \leq\left|S^{(\phi)}(E / Q)\right| \leq 2$. This completes the proof of Lemma 2 .

Theorem . Let $\mathrm{p}$ and $\mathrm{q}$ be prime satisfy $p \equiv 3(\bmod 8)$ and $q \equiv 5(\bmod 8)$, and let $\left(\frac{p}{q}\right)=-1$. If $n=p q$, then the rank of $E_{n}(Q)$ is one if and only if $C_{2 q}(Q) \neq \varnothing$.

Proof. Since the exact sequence $\left({ }^{* *}\right)$,Lemma1 and Lemma2, $E_{n}(Q)$ is one if and only if $\left|\frac{E^{\prime}(Q)}{\phi(E(Q))}\right|>1$. And $\left|\frac{E^{\prime}(Q)}{\phi(E(Q))}\right|>1$ if and only if $C_{2 q}(Q) \neq \varnothing$, since the map (***). This completes the proof of Theorem.

As we all know $C_{2 q}(Q) \neq \varnothing$ if and only if $2 q w^{2}=1+p^{2} z^{4}$ has rational solutions. And this theorem proofs $r\left(E_{n}(Q)\right) \leq 1$. The Birch Swinnerton-Dyer conjecture predicts that the rank of $E_{n}(Q)$ is non-zero with $n \equiv 5(\bmod 8)$. Then the rank of $E_{n}(Q)$ is one and the equation $2 q w^{2}=1+q^{2} z^{4}$ have rational solutions. 


\section{References}

[1] Feng K., Non-Congruent Numbers, Odd Graphs and the Birch-Swinnerton-Dyer Conjecture , Invent Math, (1987),89:527-560.

[2] Koblit W., Introduction to Elliptic Curves and Modular Forms, Springer-Verlag.(1973)

[3] R. Alter, T. B. Curtz and K. K. Kubota, Remarks and results on congruent numbers, in: Proc.3rd South Eastern Conf. Combin., Graph Theory and Compute., 1972, Florida Atlantic Univ., Boca Raton, Fla., (1972),27-35.

[4] Silverman J.H., The Arithmetic of Elliptic Curves, Springer-Verlag,.(1986) 